\title{
The Adiabatic Approximation for Quantum Spin Systems with a Spectral Gap
}

\author{
Claudio Albanese ${ }^{1,2}$ \\ 1 Institut für Theoretische Physik, ETH-Hönggerberg, CH-8093, Zürich, Switzerland \\ 2 School of Mathematics, Institute for Advanced Study, Princeton, NJ-08540, USA
}

Received: 15 January 1994 / in revised form: 15 September 1994

Summary. We estimate the accuracy of the adiabatic approximation in predicting the time evolution of local observables for an XY quantum magnet with a slowly variable external magnetic field. The system evolves according to the natural Hamiltonian dynamics and the spectral gap produced by the magnetic field is assumed to be large with respect to the term inducing quantum fluctutions. The proof is based on a finite order truncation of a time dependent cluster expansion in inverse powers of the time scale $\tau$. In the analytic case, we show that the accuracy of this truncated expansion is of order $O\left(\mathrm{e}^{-\alpha \mathrm{e} \cdot \tau^{\frac{1}{\alpha}}}\right)$ for any $\alpha>1$. If the time dependent perturbation is suddenly switched on at time zero and switched off at time $\tau$, the accuracy of the adiabatic approximation is proven to be of order $O\left(\tau^{-1}\right)$.

\section{Introduction}

The adiabatic approximation and linear response theory are basic tools of nonequilibrium statistical mechanics which are useful for setting up the memory function formalism and understanding the hydrodynamic description $[\mathrm{F}]$. In this paper, we study a quantum spin system at zero temperature for which the accuracy of these approximations can be rigorously controlled. The model is described by a time dependent Hamiltonian $\tau \mathbb{H}(t)$, where $t \in[0,1]$ is a rescaled time coordinate and $\tau \gg 1$ is the time scale. We assume that the time dependency is analytic because the study of this case appears to be more instructive. However, weaker formulations of the two results of this article hold also in case the degree of smoothness is finite. Each operator $\mathbb{H}(t)$ at fixed time $t$ has a gap of order 1 and a ground state with short range correlations which can be computed in perturbation theory. If one chooses as initial condition the instantaneous ground state at time zero, then at time $t=1$ we prove that the system is in the ground state of $\mathbb{H}(1)$ up to errors that, if measured by computing the expectation of a local observable, are of order $\tau^{-1}$. The subleading corrections can be computed in terms of an asymptotic expansion in inverse powers of $\tau$. The 
method we follow is related to the one developed by Fock [F] and refined by Kato $[\mathrm{K}]$ to prove the adiabatic theorem for one-body Schrödinger operators. The idea is to pass to a moving frame in which the generator of the dynamics annihilates the unperturbed ground state up to local corrections of order $O\left((N !)^{\alpha} \tau^{-N}\right)$. Here $\alpha$ is any constant $>1$ and $N$ is an integer $\geq 1$. The optimal value for $N$ is $\approx \mathrm{e} \tau^{\frac{1}{\alpha}}$, which corresponds to an error term of order $O\left(\mathrm{e}^{-\alpha \mathrm{e} \tau^{\frac{1}{\alpha}}}\right)$. In order to obtain bounds which are uniform in the volume, we define the moving frame by means of unitary operators with good clustering properties. This allows one to decouple the local degrees of freedom from each other. To estimate the accuracy of this truncated perturbation expansion in powers of $\tau^{-1}$, we study the generation and the propagation of disturbances in the rotating frame by means of a polymer expansion for a gas of worldlines of quasiparticle excitations.

Although the method is quite general and can accomodate a large class of models, it is useful to concentrate on a specific example. The model we consider is an anisotropic spin- $\frac{1}{2}$ antiferromagnet on the lattice $\mathbb{Z}^{d}$ with a perpendicular exchange coupling $\lambda$ which is small in absolute value. This system evolves in the presence of a variable magnetic field $\mathbf{h}(x, t)$. Here, $\mathbf{h}(x, t) \in \mathbb{R}^{d}, x \in \mathbb{Z}^{d}, t \in \mathbb{R}$, is a function of the rescaled time $t$ such that $\|\mathbf{h}(x, t)\|=1$ for all $x$ and $t$. We consider the time interval $t \in[0,1]$ and assume that $\mathbf{h}(x, t)$ admits an analytic continuation to the strip $\{\operatorname{Im} t \leq \delta\}$, for some $\delta>0$. The time scale $\tau$ on which the magnetic field varies is assumed to be large, i.e. we consider the adiabatic limit. The Hamiltonian generating the dynamics with respect to the rescaled time $t$ is $\tau \mathbb{H}(t)$, where

$$
\mathbb{H}(t)=\sum_{\langle x y\rangle \subset \mathbb{Z}^{d}} \lambda \boldsymbol{\sigma}_{x} \cdot \boldsymbol{\sigma}_{y}+\sum_{x \in \mathbb{Z}^{d}} \frac{1}{2}\left(\mathbf{h}(x, t) \cdot \boldsymbol{\sigma}_{x}+1\right) .
$$

If $\mathbb{L}$ is a local observable, then we have

$$
\langle\Psi(0)|\mathbb{U}(0,1) \mathbb{L} \mathbb{U}(1,0)| \Psi(0)\rangle=\langle\Psi(1)|\mathbb{L}| \Psi(1)\rangle+\operatorname{error}(\mathbb{L}),
$$

where $\Psi(t)$ is the instantaneous ground state of $\mathbb{H}(t)$ and $\mathbb{U}(t, 0)$ is the propagator

$$
\mathbb{U}(t, 0)=P \exp \left(i \tau \int_{0}^{t} d s \mathbb{H}(t)\right) .
$$

Theorem. There is a constant $\lambda_{0}>0$ such that if $|\lambda| \leq \lambda_{0}$ then for all local observables $\mathbb{L}$ the error term in (1.3) has an asymptotic expansion of the form

$$
\operatorname{error}(\mathbb{L}) \approx \sum_{n=1}^{\infty} a_{n}(\mathbb{L}) \tau^{-n} .
$$

The coefficients $a_{n}(\mathbb{L})$ are such that for any $\alpha>1$, there is a constant $c_{0}(\alpha, \mathbb{L}) \in(0, \infty)$ independent of the volume $|\Lambda|$ for which we have

$$
\left|a_{n}(\mathbb{L})\right| \leq c_{0}(\alpha, \mathbb{L})^{n}(n !)^{\alpha} .
$$

In general, the coefficient $a_{1}(\mathbb{L})$ is nonzero because the time interval is restricted to $[0,1]$ and the time dependent perturbation is suddenly turned on and switched off. In case the perturbation is adiabatically switched on as $t \rightarrow-\infty$ and turned off as $t \rightarrow \infty$, one would expect a much smaller error term of order $\mathrm{e}^{-c \cdot \tau}$. This delicate 
result was established by Joye and Pfister [JP] in the few body case and the extension to the many-body case might require a combination of the methods in [JP] with those in this article.

The second result concerns linear response theory. The memory function formalism is usually set up in the interaction representation [F]. Let us consider the operator

$$
\mathbb{L}(t)=\exp (i \tau t \mathbb{H}(0)) \mathbb{L} \exp (-i \tau t \mathbb{H}(0))
$$

and the density matrix $\rho(t)$ ( a simple projector since we are at zero temperature) which evolved from the time zero matrix $|\Psi(0)\rangle\langle\Psi(0)|$. The time evolution is given by the equation

$$
i \frac{d \rho(t)}{d s}=\tau \sum_{x}[\rho(t), \mathbf{M}(x, t)] \cdot \delta \mathbf{h}(x, t),
$$

where

$$
\mathbf{M}(x, t)=\exp (i \tau s \mathbb{H}(0)) \boldsymbol{\sigma}_{x} \exp (-i \tau s \mathbb{H}(0))
$$

and

$$
\delta \mathbf{h}(x, t)=\mathbf{h}(x, t)-\mathbf{h}(x, 0) .
$$

We have

$$
\begin{aligned}
& \langle\mathbb{L}(t)\rangle \equiv \operatorname{Tr}(\mathbb{L}(t) \rho(t)) \\
& =\operatorname{Tr}(\mathbb{L}(t) \rho(0))-i \tau \int_{0}^{s} d t^{\prime} \sum_{x^{\prime}} \operatorname{Tr}\left(\mathbb{L}(t)\left[\rho\left(t^{\prime}\right), \mathbf{M}\left(x^{\prime}, t^{\prime}\right)\right]\right) \cdot \delta \mathbf{h}\left(x^{\prime}, t^{\prime}\right) \\
& =\operatorname{Tr}(\mathbb{L}(t) \rho(0))-i \tau \int_{0}^{s} d t^{\prime} \sum_{x^{\prime}} \chi_{L M}\left(t, t^{\prime} ; x^{\prime}\right) \cdot \delta \mathbf{h}\left(x^{\prime}, t^{\prime}\right),
\end{aligned}
$$

where $\chi_{L M}$ is the exact response function defined as

$$
\chi_{L M}\left(t, t^{\prime} ; x^{\prime}\right)=\operatorname{Tr}\left(\rho(t)\left[\mathbb{L}_{(}(t), \mathbf{M}\left(x^{\prime}, t^{\prime}\right)\right]\right) .
$$

Corollary. Under the hypothesis of the theorem above, we have

$$
\begin{aligned}
& \chi_{L M}\left(t, t^{\prime} ; x^{\prime}\right)=\left\langle\Psi\left(t^{\prime}\right)\left|\left[\mathbb{L}(t), \mathbf{M}\left(x^{\prime}, t^{\prime}\right)\right]\right| \Psi\left(t^{\prime}\right)\right\rangle+O\left(\tau^{-1}\right) \\
& \quad=\left\langle\Psi(0)\left|\left[\mathbb{L}(t), \mathbf{M}\left(x^{\prime}, t^{\prime}\right)\right]\right| \Psi(0)\right\rangle+O\left(\tau^{-1}+\sup _{x}\left|\delta h\left(x, t^{\prime}\right)\right|\right),
\end{aligned}
$$

where the error terms depend on $\mathbb{L}$ but are uniform in the volume $|\Lambda|$.

Linear response theory is the approximation in which the error in the third term of (1.13) is neglected.

Acknowledgement. I am grateful to Tadeusz Balaban for having brought this problem to my attention.

\section{Outline of the Proof}

The classical argument to prove the adiabatic theorem due to Fock [1] and to Kato [2] is based on a time dependent change of reference frame in the Hilbert space. In 
the many body case, this idea must be implemented in a way which is consistent with the locality of the Hamiltonian and the natural clustering properties of the low energy wavefunctions. Dressing transformations [A] satisfy this requirement and are a natural tool for this kind of problem. In this paper, this technique is extended to the problem of finding approximate solutions to the time dependent Schroedinger equation for a many-body system.

The rotating reference frame is defined in several steps. In the first, we pass to the representation in which the propagator is given by the operator

$$
\mathbb{U}_{0}\left(t_{2} ; t_{1}\right)=\mathbb{B}\left(t_{2}\right)^{-1} \mathbb{U}\left(t_{2} ; t_{1}\right) \mathbb{B}\left(t_{1}\right),
$$

where $\mathbb{U}\left(t_{2} ; t_{1}\right)$ is the propagator corresponding to $\mathbb{H}(t)$ and $\mathbb{B}(t)$ is the rotation operator

$$
\mathbb{B}(t)=\prod_{x \in \Lambda} \exp \left(i(\mathbf{h}(x, t)-\mathbf{h}(x, 0)) \cdot \boldsymbol{\sigma}_{x}\right) .
$$

Let $|-\mathbf{h}\rangle_{x}$ be the state of the spin in $x$ in which the orientation is opposite to that of the magnetic field $\mathbf{h} \in \mathbb{R}^{3}$ so that we have

$$
\mathbb{B}(t) \otimes_{x \in \mathbb{Z}^{d}}|\mathbf{h}(x, 0)\rangle_{x}=\otimes_{x \in \mathbb{Z}^{d}}|\mathbf{h}(x, t)\rangle_{x} .
$$

We also have

$$
\mathbb{B}(t)^{-1} \frac{1}{2}\left(\mathbf{h}(x, t) \cdot \boldsymbol{\sigma}_{x}+1\right) \mathbb{B}(t)=\mathbb{S},
$$

where

$$
\mathbb{S}=\sum_{x \in \mathbb{Z}^{d}} s_{x}=\sum_{x \in \mathbb{Z}^{d}} \frac{1}{2}\left(\mathbf{h}(x, 0) \cdot \boldsymbol{\sigma}_{x}+1\right) .
$$

In the new representation, the dynamics'generator is given by the operator

$$
\tau \mathbb{H}_{0}(t)=\mathbb{B}(t)^{-1} \mathbb{H}(t) \mathbb{B}(t)+i \tau^{-1} \mathbb{B}^{\prime}(t) \mathbb{B}(t)^{-1}=\mathbb{S}+\lambda \mathbb{K}(t)+\tau^{-1} \mathbb{F}(t),
$$

where

$$
\mathbb{K}(t)=\sum_{\langle x y\rangle} \mathbb{B}(t)^{-1} \boldsymbol{\sigma}_{x} \cdot \boldsymbol{\sigma}_{y} \mathbb{B}(t)
$$

and

$$
\mathbb{F}(t)=\sum_{x} f_{x}=\mathbb{B}^{\prime}(t) \mathbb{B}(t)^{-1} .
$$

The goal is to solve the following equation in terms of formal power series in the inverse time scale $\tau^{-1}$ :

$$
i \frac{d}{d t} \mathrm{e}^{i \phi(t)} \mathbb{A}(t)|0\rangle=\tau \mathrm{e}^{i \phi(t)} \mathbb{H}(t) \mathbb{A}(t)|0\rangle
$$

Here, $\phi(t)$ is a phase factor with an asymptotic expansion of the form

$$
\phi(t) \approx \sum_{n=-1}^{\infty} \sum_{m=0}^{\infty} \phi_{m n}(t) \lambda^{m} \tau^{-n} .
$$

$\mathbb{A}(t)$ is a unitary operator of the form 


$$
\mathbb{A}(t)=\left(\lim _{v \rightarrow \infty} \mathrm{e}^{\mathbb{I}^{(1)}(t)} \ldots \mathrm{e}^{\mathbb{T}^{(v)}(t)}\right)
$$

defining a unitary dressing transformation. The generators $\mathbb{R}^{v}(t)$ are such that

$$
\begin{gathered}
\mathbb{R}^{(v)}(t) \approx \sum_{n=0}^{\infty} \sum_{m=1} \lambda^{m} \tau^{-n} \mathbb{R}_{m n}^{(v)}(t), \\
\mathbb{R}_{m n}^{(v)}=\sum_{|\gamma|=v} \hat{r}_{m n \gamma}(t), \\
\hat{r}_{m n \gamma}(t)=\mathscr{R}\left\{r_{m n \gamma}(t)\right\} \frac{1}{|\gamma|} \sum_{x \in \gamma} i \sigma_{x}^{(2)} \prod_{y \in \gamma \backslash\{x\}} \sigma_{y}^{(1)}+i \mathscr{T}\left\{r_{m n \gamma}(t)\right\} \prod_{x \in \gamma} \sigma_{x}^{(1)} .
\end{gathered}
$$

The function $\phi(t)$ s chosen so that $\mathbb{R}_{01}=0$. The complex valued functions $r_{m n \gamma}(t)$ are uniquelly determined by recurrence relations given in Sect. 3. $\mathscr{R}$ and $\mathscr{T}$ denote the real and imaginary parts, respectively. $\gamma$ denotes maps $: \Lambda \rightarrow\{0,1\}$ describing excitations as well as the support of such functions. The volume of $\gamma$ is $|\gamma|$, while $d(\gamma)$ denotes the number of bonds of the smallest connected cluster containing $\gamma$ as a subset. We also introduce the following distance function between two excitations $\gamma_{1}$ and $\gamma_{2}$ :

$$
d\left(\gamma_{1}, \gamma_{2}\right)=\min \left\{|\Gamma| \text { for } \Gamma \subset \Lambda \text { such that for all } x \in \gamma_{1} \backslash \gamma_{2}\right.
$$

(respectively $\gamma_{2} \backslash \gamma_{1}$ ) there is a path in $\Gamma$ joining it to $\gamma_{2}$ (respectively $\gamma_{1}$ ) $\}$.

Finally, the phase factor $\phi(t)$ is fixed by the condition (2.21) below.

To generate the asymptotic expansion in powers of $\tau^{-1}$, we pass to the reference frame in which the propagator is given by the operator

$$
\mathbb{U}_{1}\left(t_{2} ; t_{1}\right)=\mathrm{e}^{i\left(\phi\left(t_{1}\right)-\phi\left(t_{2}\right)\right)} \mathbb{A}\left(t_{2}\right)^{-1} \mathbb{U}_{0}\left(t_{2} ; t_{1}\right) \mathbb{A}\left(t_{1}\right) .
$$

In this moving coordinate system, the time dependent Schroedinger equation has the form

$$
i \frac{d}{d t} \mathbb{U}_{0}\left(t_{2} ; t_{1}\right)=-\tau \mathbb{U}_{0}\left(t_{2} ; t_{1}\right) \mathbb{H}_{0}\left(t_{1}\right),
$$

where

$$
\mathbb{H}_{D}(t)=\mathbb{A}(t)^{-1} \mathbb{H}_{0}(t) \mathbb{A}(t)+i \tau^{-1} \mathbb{A}(t)^{-1} \mathbb{A}^{\prime}(t)+i \tau^{-1} \phi^{\prime}(t) .
$$

The first $N$ terms of the formal $\frac{1}{\tau}$ expansion is generated by requiring that

$$
\mathbb{H}_{D}(t)|0\rangle=\mathbb{Z}_{N}(t)|0\rangle,
$$

where $\mathbb{Z}_{N}(t)$ is a local operator whose off diagonal part is of order $O\left(\tau^{-N}\right)$ while the coefficients in the expansion (2.10) for the phase factor $\phi(t)$ are fixed by the condition

$$
\left\langle 0\left|\mathbb{Z}_{N}(t)\right| 0\right\rangle=0 .
$$

Definition. If $a, b \in \mathbb{R}$, let $a \cup b=\max (a, b)$ and $a \cap b=\min (a, b)$.

In the next section we prove the following result: 
Lemma. For all $N \geq 1$ there is a transformation $\mathbb{A}_{N}(t)$ of the form (2.11) such that if $\mathbb{L}$ is a local operator and

$$
\mathbb{L}_{N}(t) \equiv \mathbb{A}_{N}(t)^{-1} \mathbb{L} \mathbb{A}_{N}(t)=\sum_{n=0}^{\infty} \sum_{m=0}^{\infty} \tau^{-n} \lambda^{m} \sum_{\gamma \subset \Lambda} l_{N n m}(t, \gamma)
$$

is a decomposition of the dressed operator $\mathbb{L}_{N}(t)$ into operators $l_{N n m}(t ; \gamma)$ of support $\gamma$, we have that

$$
\sum_{d(\gamma) \geq d_{0}}\left\|l_{D n}(t ; \gamma)\right\|_{1} \leq\|\mathbb{L}\|_{1} c^{m} c(\alpha)^{n}(n !)^{\alpha}(c \cdot \lambda)^{\left(d_{0}-n\right) \cup 1}
$$

for all constants $\alpha>1$ and some constant $c, c(\alpha)>0$ which do not depend on $n$. Moreover, the dressed Hamiltonian has the form

$$
\mathbb{H}_{N}(t)=\mathbb{S}+\mathbb{V}(t)+\mathbb{Z}_{N}(t)
$$

where

$$
\mathbb{V}(t)=\sum_{n=0}^{N} \sum_{m=1}^{\infty} \sum_{\gamma \subset \Lambda} \tau^{-n} \lambda^{m} v_{n m}(t ; \gamma),
$$

and the operators $v_{n m}(t ; \gamma)$ have support $\gamma$ and are such that

$$
\sum_{\gamma}\left\|v_{n m}(t ; \gamma)\right\|_{1} \leq c^{m} c(\alpha)^{n}((n \cap N) !)^{\alpha}
$$

and

$$
v_{n m}(t ; \gamma)|0\rangle=0
$$

Finally, the operator $\mathbb{Z}_{N}(t)$ admits a decomposition into local operators

$$
\mathbb{Z}_{N}(t)=\sum_{\gamma \subset \Lambda} z_{N}(t ; \gamma)
$$

with $\operatorname{supp} z_{N}(t ; \gamma) \subset \gamma$, such that

$$
\sum_{d(\gamma) \geq d_{0}}\left\|z_{N}(t ; \gamma)\right\|_{1} \leq(N !)^{\alpha} \tau^{-N}(c \cdot \lambda)^{\left(d_{0}-N\right) \cup 1}
$$

Since the expansion in inverse powers of $\tau$ does not converge in the limit $N \rightarrow \infty$, it is worth computing only a number of terms $\approx \mathrm{e} \tau^{\frac{1}{\alpha}}$, after which the addition of higher order terms worsens, rather than improves, the accuracy of the approximation. We thus need a separate argument to estimate the accuracy of our time dependent cluster expansion, truncated at finite order in $\frac{1}{\tau}$. To this end, we make use of the linked cluster theorem [GJ].

Let $\mathbb{A}_{\infty}(t)$ denote the unitary operator in (2.11) in the limit $\tau=\infty$. The error term in (1.5) is given by

$$
\begin{aligned}
\operatorname{error}(\mathbb{L}) & =\left\langle 0\left|\mathbb{A}_{\infty}(0)^{-1} \mathbb{U}_{0}(0 ; 1) \mathbb{L}_{0}(1 ; 0) \mathbb{A}_{\infty}(0)\right| 0\right\rangle-\left\langle 0\left|\mathbb{A}_{\infty}(1)^{-1} \mathbb{L} \mathbb{A}_{\infty}(1)\right| 0\right\rangle \\
& =\left\langle 0\left|\mathbb{U}_{1}(0 ; 1) \mathbb{A}(1)^{-1} \mathbb{L} \mathbb{A}(1) \mathbb{U}_{1}(1 ; 0)\right| 0\right\rangle-\left\langle 0\left|\mathbb{A}_{\infty}(1)^{-1} \mathbb{L} \mathbb{A}_{\infty}(1)\right| 0\right\rangle .
\end{aligned}
$$


Here, we make use of the fact that, thanks to the initial condition at $t=0$, the vector $\mathbb{A}_{\infty}(0)|0\rangle$ is the ground state of $\mathbb{H}_{0}(0)$. Let $l_{n}(\lambda, \gamma)$ and $l_{\infty n}(\lambda, \gamma)$ be the families of operators of support $\gamma$ such that

$$
\mathbb{A}(1)^{-1} \mathbb{L} \mathbb{A}(1)=\sum_{n=0}^{N} \tau^{-n} \sum_{\gamma} l_{n}(\lambda, \gamma)
$$

and

$$
\mathbb{A}_{\infty}(1)^{-1} \mathbb{L}_{\mathbb{A}_{\infty}}(1)=\sum_{n=0}^{N} \tau^{-n} \sum_{\gamma} l_{\infty n}(\lambda, \gamma)
$$

respectively, and let

$$
\bar{l}_{n}(\lambda, \gamma)=l_{n}(\lambda, \gamma)-\left\langle 0\left|l_{\infty, n}(\lambda, \gamma)\right| 0\right\rangle .
$$

Equation (2.30) can be written as follows:

$$
\operatorname{error}(\mathbb{L})=\sum_{n=0}^{N} \tau^{-n} \sum_{\gamma}\left\langle 0\left|\mathbb{U}_{1}(0 ; 1) \bar{l}_{n}(\lambda, \gamma) \mathbb{U}_{1}(1 ; 0)\right| 0\right\rangle .
$$

Thanks to the lemma above, we have that

$$
\left|\left\langle 0\left|\bar{l}_{n}(\lambda, \gamma)\right| 0\right\rangle\right| \leq(n !)^{\alpha} c(\alpha)^{n}(c \cdot \lambda)^{(d(\gamma)-n) \cup 1} \cdot \tau^{-n} .
$$

We also know the relative boundedness estimate

$$
\langle\Psi|(\bar{l}(\lambda, \gamma)-\langle 0|\bar{l}(\lambda, \gamma)| 0\rangle)| \Psi\rangle \leq\|\bar{l}(\lambda, \gamma)\|_{2}\left\langle\Psi\left|\sum_{x \in \gamma} s_{x}\right| \Psi\right\rangle
$$

where $s_{x}$ is the operator in (2.5). Hence, it suffices to prove that in the rotating reference frame we have:

$$
\sup _{x \in \Lambda}\left\langle 0\left|\mathbb{U}_{0}(0 ; 1) s_{x} \mathbb{U}_{0}(1 ; 0)\right| 0\right\rangle=\sum_{n=0}^{N} a_{n}\left(\lambda, s_{x}\right) \tau^{-n},
$$

where the coefficients $a_{n}\left(s_{x}\right)$ satisfy the following estimate:

$$
a_{n}\left(s_{x}\right) \leq c(\alpha)^{n} \cdot(n !)^{\alpha} .
$$

To this end, we can use a polymer expansion of the type in [GJ].

We call a (simple) polymer a map $\bar{\gamma}(t)$ defined for $t \in[0,1]$. We assume that the range of values of a polymer $\bar{\gamma}$ consists of a finite number $n(\bar{\gamma})$ of different subsets of $\Lambda$. We also assume that $\bar{\gamma}(t)$ is upper semicontinuous so that if $t_{0}, \ldots t_{n(\bar{\gamma})}$ are the points of discontinuity of the function $\bar{\gamma}$, then $\bar{\gamma}(t)$ is constant on the intervals $\left[t_{i}, t_{i+1}\right), i=0, \ldots n(\bar{\gamma})-1$. Finally, we assume that the graph of the function $\bar{\gamma}$, i.e. the set $\{(t, \bar{\gamma}(t)), t \in[0,1]\} \subset \mathbb{R} \times \Lambda$, is connected. The support of $\bar{\gamma}$ is the set supp $\bar{\gamma} \equiv \cup_{i=1}^{n(\bar{\gamma})} \bar{\gamma}\left(t_{i}\right)$ and the range is $\operatorname{Ran} \bar{\gamma}=\left\{\bar{\gamma}\left(t_{1}\right), \cdots \bar{\gamma}\left(t_{n(\bar{\gamma})}\right)\right\}$. Following [GJ], let us denote by $\mathscr{P}_{1}$ the set of (simple) polymers and by $\mathscr{T}_{k}$ the set of $k$-polymers, i.e. the $k$-fold product $\mathscr{P}_{k}=\mathscr{P}_{1} \times \ldots \times \mathscr{P}_{1}$.

Two polymers $\bar{\gamma}_{1}$ and $\bar{\gamma}_{2}$ are said to intersect if 


$$
\operatorname{supp} \bar{\gamma}_{1} \cap \operatorname{supp} \bar{\gamma}_{2} \neq \emptyset \text {. }
$$

In this case, we write $\bar{\gamma}_{1} \cap \bar{\gamma}_{2} \neq \emptyset$. Otherwise, we say that $\bar{\gamma}_{1}$ and $\bar{\gamma}_{2}$ do not intersect and we write $\bar{\gamma}_{1} \cap \bar{\gamma}_{2}=\emptyset$. Let $\mathscr{V}_{k}$ and $\mathscr{C}_{k}$ denote the sets of the disjoint and of the connected $k$-polymers in $\mathscr{P}_{k}$, respectively.

The wavefunction $\mathbb{U}_{1}(1 ; 0)|0\rangle$ admits a polymer expansion of the following form:

$$
\mathbb{U}_{1}(1 ; 0)|0\rangle=\sum_{\jmath=0}^{\infty} \frac{1}{j !} \int_{\mathscr{U}_{\jmath}}\left[d \bar{\gamma}_{1}\right] \ldots\left[d \bar{\gamma}_{j}\right] \psi\left(\bar{\gamma}_{1}\right) \ldots \psi\left(\bar{\gamma}_{j}\right)\left|\cup_{k=1}^{j} \bar{\gamma}_{j}(1)\right\rangle,
$$

where the functional integral is defined in such a way that

$$
\begin{aligned}
& \int_{\mathscr{V}_{j}}\left[d \bar{\gamma}_{1}\right] \cdots\left[d \bar{\gamma}_{j}\right] \psi\left(\bar{\gamma}_{1}\right) \cdots \psi\left(\bar{\gamma}_{\jmath}\right) \\
& =\sum_{\quad \operatorname{supp}_{\left.\left(\bar{\gamma}_{1} \cdots \operatorname{\gamma upp}_{1}, \ldots \bar{\gamma}_{j}\right) \in \mathscr{V}_{j}\right)}} \int_{0}^{1} d t_{1} \cdots \int_{0}^{t_{\bar{N}-1}} d t_{\bar{N}} \\
& \cdot T \prod_{k=1}^{j} \psi\left(\gamma_{k 1}, \cdots \gamma_{k n\left(\gamma_{k}\right)} ; t_{1}, \cdots t_{n\left(\gamma_{k}\right)-1}\right) .
\end{aligned}
$$

Here, $T$ is the time ordering operator and $\bar{N}=\sum_{k=1}^{j} n\left(\gamma_{k}\right)$. We also set

$$
\begin{aligned}
\psi\left(\gamma_{1}, \cdots \gamma_{n} ; t_{1}, \cdots t_{n}\right) & \\
=\mathrm{i}^{n}\left\langle\gamma_{n}\right| & \prod_{j=n-1, \ldots 1}\left[\mathrm{e}^{-i \tau\left(t_{j+1}-t_{j}\right) \mathbb{S}}\left(v\left(t_{j} ; \gamma_{j}\right)+z_{N}\left(t_{j} ; \gamma_{j}\right)\right)\right]|0\rangle,
\end{aligned}
$$

where $t_{n+1}=1$ and the factors in the product are arranged in such a way to show decreasing values of $j$ as one goes from the left to the right.

To show how to estimate functional integrals of this type, let us prove the following upper bound:

$$
\sup _{x \in \Lambda}\left|\int_{\mathscr{P}\left(\gamma, d_{0}\right)}[d \bar{\gamma}] \psi(\bar{\gamma})\right| \leq(N !)^{\alpha} c(\alpha)^{N} \tau^{-N}(c \cdot \lambda)^{d_{0}},
$$

where $\mathscr{P}\left(\gamma, d_{0}\right)$ is the set of all simple polymers $\bar{\gamma}$ such that $\bar{\gamma}\left(t_{1}\right)=\gamma$ and

$$
d_{0}=d(\gamma, \operatorname{supp} \bar{\gamma})
$$

Integrating by parts, we find that

$$
\int_{0}^{t_{2}} \mathrm{e}^{-i \tau\left(t_{1}-t_{2}\right) \mathbb{S}} z_{N}\left(t_{1} ; \gamma_{1}\right)=\frac{\mathrm{i}}{\tau \mathbb{S}} \mathbb{W}_{1}\left(t_{2} ; \gamma_{1}\right),
$$

where

$$
\mathbb{W}_{1}\left(t_{2} ; \gamma_{1}\right)=\left(\mathbb{I}-\mathrm{e}^{i \tau t_{2} \mathbb{S}}\right) z_{N}\left(t_{2} ; \gamma_{1}\right)-\int_{0}^{t_{2}} d t_{1} \mathrm{e}^{\imath \tau\left(t_{2}-t_{1}\right) \mathbb{S}} z_{N}^{\prime}\left(t_{1} ; \gamma_{1}\right)
$$

Similarly, if $j=2, \ldots n$, we find that 


$$
\begin{aligned}
& \left(\frac{\mathrm{i}}{\tau}\right)^{j-1} \int_{0}^{\tau_{\jmath+1}} d t_{j} \mathrm{e}^{-i \tau\left(t_{\jmath}-t_{\jmath+1}\right) \mathbb{S}}\left(v\left(t_{j} ; \gamma_{j}\right)+z_{N}\left(t_{j} ; \gamma_{j}\right)\right) \\
& \cdot \mathbb{S}^{-1} \mathbb{W}_{j-1}\left(t_{j} ; \gamma_{1}, \cdots \gamma_{j-1}\right)=\left(\frac{\mathrm{i}}{\tau}\right)^{j} \mathbb{S}^{-1} \mathbb{W}_{j}\left(t_{j+1} ; \gamma_{1}, \cdots \gamma_{j}\right)
\end{aligned}
$$

where

$$
\begin{aligned}
& \mathbb{W}_{\jmath}\left(t_{j+1} ; \gamma_{1}, \ldots \gamma_{j}\right) \\
& =\left(\mathbb{I}-\mathrm{e}^{\imath \tau t_{j+1} \mathbb{S}}\right)\left(v\left(t_{j+1} ; \gamma_{j}\right)+z_{N}\left(t_{j+1} ; \gamma_{\jmath}\right)\right) \mathbb{S}^{-1} \mathbb{W}_{j-1}\left(t_{\jmath+1} ; \gamma_{1}, \ldots \gamma_{j-1}\right) \\
& \quad-\int_{0}^{t_{\jmath+1}} d t_{j} \mathrm{e}^{i \tau\left(t_{j+1}-t_{\jmath}\right) \mathbb{S}} \\
& \quad \frac{d}{d t_{\jmath}}\left[\left(v\left(t_{j} ; \gamma_{j}\right)+z_{N}\left(t_{j} ; \gamma_{j}\right)\right) \mathbb{S}^{-1} \mathbb{W}_{j-1}\left(t_{j} ; \gamma_{1}, \cdots \gamma_{\jmath-1}\right)\right]
\end{aligned}
$$

Finally, we have that

$$
\int_{\mathscr{P}\left(\gamma, d_{0}\right)}[d \bar{\gamma}] \psi(\bar{\gamma})=\sum_{\substack{\left(\gamma_{1}, \ldots \gamma_{n}\right)=\operatorname{Ran} \bar{\gamma}: \\ \bar{\gamma} \in \mathscr{P}\left(\gamma, d_{0}\right)}}\left\langle\gamma_{n}\left|\mathbb{W}_{n-1}\left(1 ; \gamma_{1}, \ldots \gamma_{n-1}\right)\right| 0\right\rangle .
$$

Let $\mathbb{W}_{j}^{(0)}\left(t_{j+1} ; \gamma_{1}, \ldots \gamma_{j}\right), j=1, \ldots . . n$, be the operators corresponding to polymers with only one insertion of an operator $z_{n}$ at time $t=t_{1}$, i.e. the operators such that $\mathbb{W W}_{1}^{(0)}\left(t_{2} ; \gamma_{1}\right)=\mathbb{W}_{1}^{(1)}\left(t_{2} ; \gamma_{1}\right)$ and such that if $j \geq 2$ then

$$
\begin{gathered}
\mathbb{W}_{j}^{(0)}\left(t_{j+1} ; \gamma_{1}, \cdots \gamma_{\jmath}\right)=\left(\mathbb{I}-\mathrm{e}^{i \tau t_{j+1} \mathbb{S}}\right) v\left(t_{\jmath+1} ; \gamma_{j}\right) \mathbb{S}^{-1} \mathbb{W}_{\jmath-1}\left(t_{j} ; \gamma_{1}, \cdots \gamma_{j-1}\right) \\
-\int_{0}^{t_{j+1}} d t_{j} \mathrm{e}^{i \tau\left(t_{j+1}-t_{j}\right) \mathbb{S}} \frac{d}{d t_{j}}\left[v\left(t_{j} ; \gamma_{j}\right) \mathbb{S}^{-1} \mathbb{W W}_{j-1}^{(0)}\left(t_{j} ; \gamma_{1}, \cdots \gamma_{j-1}\right)\right]
\end{gathered}
$$

If

$$
v_{m}(t ; \gamma)=\sum_{n=0}^{N} \tau^{-n} v_{n m}(t ; \gamma)
$$

and

$$
\mathbb{W}_{2}^{(0)}\left(t ; \gamma_{1}, \gamma_{2}\right)|0\rangle=\sum_{\gamma_{0}} w_{2}^{(0)}\left(t ; \gamma_{1}, \gamma_{2} ; \gamma_{0}\right) \tau_{\gamma_{0}}|0\rangle
$$

we have

$$
\begin{aligned}
\sup _{x} & \sum_{\substack{d\left(\gamma_{0}\right) \geq d_{0} \\
\gamma_{0} \ni x}} \sum_{\gamma_{1}, \gamma_{2}}\left|w_{2}^{(0)}\left(t ; \gamma_{1}, \gamma_{2} ; \gamma_{0}\right)\right| \\
\leq & \sum_{m=1}^{\infty} \lambda^{m} \sum_{\gamma_{0}^{\prime}:\left|\gamma_{0}^{\prime}\right| \geq d_{0}-m} \frac{1}{\left|\gamma_{0}^{\prime}\right|} \sum_{y \in \gamma_{0}^{\prime}} \sum_{y_{2} \ni y}\left\|v_{m}\left(t ; \gamma_{2}\right)\right\|_{1} \sum_{\gamma_{1}}\left|w_{1}^{(0)}\left(t ; \gamma_{1} ; \gamma_{0}^{\prime}\right)\right| \\
\leq & (N !)^{\alpha} c(\alpha)^{N} \tau^{-N}(c \cdot \lambda)^{d_{0}}
\end{aligned}
$$

Here, the key remark is that the operators $v_{m}\left(t ; \gamma_{0}\right)$ annihilate the states in the Ising basis for which the set $\gamma_{2}$ does not contain excitations. The volume factor arising 
from the sum over the base point $y \in \gamma_{0}^{\prime}$ is canceled by the factor coming from the operator $\mathbb{S}^{-1}$.

To obtain (2.43), the estimates above can be iterated over $j$ up to $j=n-1$. It is not difficult to convince oneselves that the qualitative form of these estimates is preserved if one allows multiple insertions of operators $z_{N}$.

To find a polymer expansion for the expectation value of a local observable $\mathbb{L}$, we have to slightly modify the notion of intersection between two polymers. We say that $\bar{\gamma}_{1}$ intersects $\bar{\gamma}_{2}$ modulo $\mathbb{L}$ and write $\bar{\gamma}_{1} \cap_{\mathbb{L}} \bar{\gamma}_{2} \neq \emptyset$, if either $\bar{\gamma}_{1} \cap \bar{\gamma}_{2} \neq \emptyset$ or if supp $\bar{\gamma}_{1}$ and supp $\bar{\gamma}_{2}$ both intersect the support of $\mathbb{L}$. Given this notion of intersection, the sets $\mathscr{D}_{j}(\mathbb{L})$ and $\mathscr{C}_{j}(\mathbb{L})$ are defined as usual. We have that

$$
\left\langle 0\left|\mathbb{U}_{1}(0 ; 1) \mathbb{L} \mathbb{U}_{1}(1 ; 0)\right| 0\right\rangle=\langle 0|\mathbb{L}| 0\rangle+\sum_{j=0}^{\infty} \frac{1}{j !} \int_{\mathscr{D}_{\jmath}(\mathbb{L})}\left[d \bar{\gamma}_{1}\right] \ldots\left[d \bar{\gamma}_{j}\right] w_{\mathbb{L}}\left(\bar{\gamma}_{1}\right) \ldots w_{\mathbb{L}}\left(\bar{\gamma}_{j}\right)
$$

where

$$
w_{\mathbb{L}}(\bar{\gamma})=\left\{\begin{array}{lc}
|\psi(\bar{\gamma})|^{2} & \text { if } \bar{\gamma}(1) \cap \operatorname{supp} \mathbb{L}=\emptyset \\
|\psi(\bar{\gamma})|^{2}\langle\bar{\gamma}(1)|\mathbb{L}| \bar{\gamma}(1)\rangle & \text { otherwise }
\end{array}\right.
$$

Also in this case, a bound similar to (2.53) holds, i.e.

$$
w(\mathbb{L} ; \gamma) \leq c(\mathbb{L}, \alpha)^{N}(N !)^{2 \alpha} \tau^{-2 N}(c \cdot \lambda)^{2 d(\gamma)} .
$$

Thanks to the linked cluster theorem, we have that

$$
\begin{aligned}
& \left\langle 0\left|\mathbb{U}_{1}(0 ; 1) \mathbb{L} \mathbb{U}_{1}(1 ; 0)\right| 0\right\rangle=-1+\langle 0|\mathbb{L}| 0\rangle \\
& \quad+\exp \left(\sum_{j=1}^{\infty} \frac{1}{j !} \int_{\mathscr{C}_{j}(\mathbb{L})}\left[d \bar{\gamma}_{1}\right] \cdots\left[d \bar{\gamma}_{j}\right] n\left(\bar{\gamma}_{1}, \cdots \bar{\gamma}_{j}\right) w_{\mathbb{L}}\left(\bar{\gamma}_{1}\right) \cdots w_{\mathbb{L}}\left(\bar{\gamma}_{j}\right)\right),
\end{aligned}
$$

where $n\left(\bar{\gamma}_{1}, \cdots \bar{\gamma}_{j}\right) \in \mathscr{C}_{j}(\mathbb{L})$ is the index of the connected polymer $\left(\bar{\gamma}_{1}, \ldots \bar{\gamma}_{j}\right)$, see [GJ]. Thanks to the exponential decay estimate (2.56), the expansion converges [GJ]. Since $\mathbb{U}_{0}(0 ; 1)$ is unitary, we also find

$$
\sum_{j=1}^{\infty} \frac{1}{j !} \int_{\mathscr{C}_{j}}\left[d \bar{\gamma}_{1}\right] \ldots\left[d \bar{\gamma}_{j}\right] n\left(\bar{\gamma}_{1}, \ldots \bar{\gamma}_{j}\right) w_{\mathbb{I}}\left(\bar{\gamma}_{1}\right) \ldots w_{\mathbb{I}}\left(\bar{\gamma}_{j}\right)=0
$$

where $\mathbb{I}$ is the identity operator. Hence

$$
\begin{aligned}
& \left\langle 0\left|\mathbb{U}_{1}(0 ; 1) \mathbb{L} \mathbb{U}_{1}(1 ; 0)\right| 0\right\rangle=-1+\langle 0|\mathbb{L}| 0\rangle \\
& \quad+\exp \left(\sum _ { j = 1 } ^ { \infty } \frac { 1 } { j ! } \left({ }_{\mathscr{C}_{j}(\mathbb{L}) \cap \operatorname{supp}(\mathbb{L}) \neq \emptyset}\left[d \bar{\gamma}_{1}\right] \cdots\left[d \bar{\gamma}_{j}\right] n\left(\bar{\gamma}_{1}, \cdots \bar{\gamma}_{j}\right) w_{\mathbb{L}}\left(\bar{\gamma}_{1}\right) \cdots w_{\mathbb{L}}\left(\bar{\gamma}_{j}\right)\right.\right. \\
& \left.\left.\quad-\int_{\mathscr{C}_{J}(\mathbb{L}) \cap \operatorname{supp}(\mathbb{L}) \neq \emptyset}\left[d \bar{\gamma}_{1}\right] \cdots\left[d \bar{\gamma}_{j}\right] n\left(\bar{\gamma}_{1}, \cdots \bar{\gamma}_{j}\right) w_{\mathbb{I}}\left(\bar{\gamma}_{1}\right) \cdots w_{\mathbb{I}}\left(\bar{\gamma}_{j}\right)\right)\right) .
\end{aligned}
$$

If $X \in \mathscr{C}(\Lambda)$ doesn't intersect the support of $\mathbb{L}$, we have that $w(\mathbb{L})^{X}=w(\mathbb{I})^{X}$ so that the summation in (2.59) can be restricted to the connected polymers intersecting supp ( $\mathbb{L}$ ). Specializing to $\mathbb{L}=s_{x}$ and using the bound (2.41) and the methods in 
[GJ], one obtains the bound in (2.38). To complete the proof, in the next section we construct the operators $\mathbb{A}(t)$ in $(2.11)$ and prove the lemma above.

\section{The Time Dependent Dressing Transformation}

In this section, we generate a formal cluster expansion in powers of $\lambda$ and $\tau^{-1}$ for a unitary operator $\mathbb{A}(t)$ of the form (2.11) which solves the following time dependent equation:

$$
\left[\mathbb{A}(t)^{-1} \mathbb{H}_{0}(t) \mathbb{A}(t)+i \tau^{-1} \mathbb{A}(t)^{-1} \mathbb{A}^{\prime}(t)+i \tau^{-1} \phi^{\prime}(t)\right]|0\rangle=0 .
$$

The expansion has the form of a double power series that converges in $\lambda$ and is asymptotic in $\tau^{-1}$. The operators $\mathbb{R}_{n m}^{v}$ satisfying (3.1) at least in the sense of formal power series are uniquely determined by the following recurrence relations:

$$
\begin{aligned}
& \sum_{v} \mathbb{R}_{n m}^{v} \mid 0> \\
& =-\left\{\sum_{x} \sum_{k \geq 2} \sum_{\left|\gamma_{1}\right| \leq \ldots \leq\left|\gamma_{k}\right|} \sum_{n_{1}+. .+n_{k}=n} \frac{1}{n\left(\left|\gamma_{1}\right|, \ldots\left|\gamma_{k}\right|\right)} \mathbb{S}^{-1} \mathbb{P}_{\perp}\right. \\
& m_{1}+\ldots+m_{k}=m \\
& {\left[\ldots\left[s_{x}, \hat{r}_{m_{1} n_{1} \gamma_{1}}(t)\right], \ldots \hat{r}_{m_{k} n_{k} \gamma_{k}}(t)\right]|0\rangle} \\
& +\sum_{\langle x y\rangle} \sum_{k \geq 0} \sum_{\left|\gamma_{1}\right| \leq \ldots \leq\left|\gamma_{k}\right|} \sum_{n_{1}+\ldots+n_{k}=n} \frac{1}{n\left(\left|\gamma_{1}\right|, \ldots\left|\gamma_{k}\right|\right)} \mathbb{S}^{-1} \mathbb{P}_{\perp} \\
& m_{1}+\ldots+m_{k}=m-1 \\
& {\left[\ldots\left[k_{\langle x y\rangle}(t), \hat{r}_{m_{1} n_{1} \gamma_{1}}(t)\right], \ldots \hat{r}_{m_{k} n_{k} \gamma_{k}}(t)\right]|0\rangle} \\
& +\sum_{x} \sum_{k \geq 0} \sum_{\substack{\left|\gamma_{1}\right| \leq \ldots \leq\left|\gamma_{k}\right| \\
n_{1}+\ldots+n_{k}=n-1 \\
m_{1}+\ldots+m_{k}=m}} \frac{1}{n\left(\left|\gamma_{1}\right|, \ldots\left|\gamma_{k}\right|\right)} \mathbb{S}^{-1} \mathbb{P}_{\perp} \\
& {\left[\cdots\left[f_{x}(t), \hat{r}_{m_{1} n_{1} \gamma_{1}}(t)\right], \ldots \hat{r}_{m_{k} n_{k} \gamma_{k}}(t)\right]|0\rangle} \\
& +\sum_{k \geq 0} \sum_{\left|\gamma_{0}\right| \leq . . \leq\left|\gamma_{k}\right|} \sum_{m_{1}+\ldots+m_{k}=m} \frac{\mathrm{i}}{n\left(\left|\gamma_{1}\right|, \ldots\left|\gamma_{k}\right|\right)} \mathbb{S}^{-1} \mathbb{P}_{\perp} \\
& n_{1}+\ldots+n_{k}=n-1 \\
& \left.\left[\ldots\left[\hat{r}_{m_{0} n_{0} \gamma_{0}}^{\prime}(t), \hat{r}_{m_{1} n_{1} \gamma_{1}}(t)\right], \ldots \hat{r}_{m_{k} n_{k} \gamma_{k}}(t)\right]|0\rangle\right\} \text {, }
\end{aligned}
$$

where $\mathbb{S}$ is defined in (2.5), $\mathbb{P}_{\perp}=\mathbb{I}-|0\rangle\langle 0|$ and we set

$$
n\left(\left|\gamma_{1}\right|, \ldots\left|\gamma_{k}\right|\right)=\prod_{v=0}^{\infty}\left(\#\left\{i:\left|\gamma_{i}\right|=v\right\} !\right)
$$


Equation (3.2) generates a cluster expansion. In fact, at the $(n+m)^{\text {th }}$ order of perturbation theory, only clusters of at most $(n+m+1)$ sites are present.

Let us introduce the following functions:

$$
r_{n m}^{*}(t)=\frac{1}{(m !)^{\alpha}} \sup _{x} \sum_{\gamma \ni x} \sup _{\mid \operatorname{Im}}\left\|r_{m n \gamma}(t)\right\|_{1} .
$$

Here, $\alpha>1$ and $\delta_{m}$ is the decreasing sequence of positive numbers such that

$$
\delta_{m}=\delta_{m-1}-\delta_{0} m^{-\alpha} c(\alpha) .
$$

$\delta_{0}$ is a positive small constant we fix below and

$$
c(\alpha)=\left(\sum_{m=1}^{\infty} m^{-\alpha}\right)^{-1} .
$$

In the following, we show that if $|\lambda|$ and $\tau^{-1}$ are small enough, we have

$$
r^{*}(t ; \lambda, \tau) \equiv \sum_{m n} r_{m n}^{*} \lambda^{m} \tau^{-m}<\infty
$$

The lemma in the previous section is a straightforward consequence of this estimate.

The first three terms in (3.2) are also present in the static, i.e. $\tau=\infty$, case and can be estimated as in $[\mathrm{A}]$ or in $[\mathrm{AF}]$. The fourth term is of a different nature because of the presence of a time derivative in the function $r_{m_{0} n_{0} \gamma_{0}}^{\prime}(t)$. To estimate its size, one can use Cauchy's theorem according to which if $m>m_{0}$ we have

$$
\begin{aligned}
\sup _{|\operatorname{Im} \delta| \leq \delta_{m}}\left\|r_{m_{0} n_{0} \gamma_{0}}^{\prime}(t)\right\|_{1} & \leq\left(\delta_{m_{0}}-\delta_{m}\right)^{-1} \sup _{|\operatorname{Im} s| \leq \delta_{m_{0}}}\left\|r_{m_{0} n_{0} \gamma_{0}}(t)\right\|_{1} \\
& \leq\left(m_{0}+1\right)^{\alpha} \sup _{|\operatorname{Im} s| \leq \delta_{m_{0}}}\left\|r_{m_{0} n_{0} \gamma_{0}}(t)\right\|_{1} .
\end{aligned}
$$

Hence, the $k=0$ contribution to $r_{m n}^{*}$ coming from the fourth term in (3.2) is bounded from above by $r_{m-1, n}^{*}$. If $k \geq 1$, we have to estimate also the commutators. Let us call $k$-polymer a family of clusters $p=\left\{\gamma_{0}, \ldots \gamma_{k}\right\}$ such that $\gamma_{0} \cap \gamma_{1} \neq \emptyset, \gamma_{k} \cap\left(\gamma_{0} \cup\right.$ $\left.\ldots \cup \gamma_{k-1}\right) \neq \emptyset$ and $\left|\gamma_{0}\right| \leq \ldots \leq\left|\gamma_{k}\right|$. Let us introduce also the equivalence class $[p]=\left\{\mathscr{T}_{x} p ; x \in \Lambda\right\}$, where $x \in \bar{\Lambda}$ and $\mathscr{T}_{x}$ is the operator of translation by $x$. There is a state $|\bar{\gamma}(p)\rangle$ such that

$$
\left.\left[. . \hat{\epsilon}_{x}, \hat{r}_{m_{1} n_{1} \gamma_{1}}^{\prime}(t)\right], \ldots \hat{r}_{m_{k} n_{k} \gamma_{k}} \tau_{\gamma_{k}}(t)\right]|0\rangle=\tilde{f}_{(m)(n)}(p, t)|\bar{\gamma}(p, t)\rangle,
$$

for some coefficient $f_{(m)(n)}(p, t)$, where $(m)=\left(m_{1} \ldots m_{k}\right),(n)=\left(n_{1} \ldots n_{k}\right)$. Let $f(p)$ be the translation invariant function such that

$$
f_{(m)(n)}(p)=\sup _{x \in \Lambda}\left|\tilde{f}_{(m)(n)}\left(\mathscr{T}_{x} p, t\right)\right| .
$$

The contribution to $r_{n}^{*}$ coming from all connected $k$-polymers $p \in[p]$ is bounded from above by 


$$
\begin{aligned}
& \left.\sup _{\substack{x \in \Lambda \\
t \in[0,1]}} \sum_{\substack{p^{\prime} \in[p] \\
\bar{\gamma}\left(p^{\prime}\right) \ni x}}\left|\tilde{f}_{(m)(n)}\left(p^{\prime}\right) \| \mathbb{S}^{-1}\right| \bar{\gamma}\left(p^{\prime}\right)\right\rangle \|_{1} \\
& \\
& \quad \leq|\bar{\gamma}(p)|\left|f_{(m)(n)}(p)\right| \| \mathbb{S}^{-1}|\bar{\gamma}(p)\rangle \|_{1}=\left|f_{(m)(n)}(p)\right| .
\end{aligned}
$$

Hence, the contribution to $r_{n}^{*}$ coming from the fourth sum in (3.2) is

$$
\begin{aligned}
& \leq \sum_{[p]} \sum_{\begin{array}{c}
k \geq 0 \\
n_{1}+\cdots+n_{k}=n-1 \\
m_{1}+\cdots+m_{k}=m
\end{array}} \frac{1}{n(p)}\left|f_{(m)(n)}(p(\pi))\right| \\
& \leq \sup _{x} \sum_{k \geq 0} \sum_{\substack{\gamma_{1}, \cdots, \gamma_{k} \\
n_{1}+\cdots+n_{k}=n \\
m_{1}+\cdots+m_{k}=m-1}} \frac{1}{n\left(\gamma_{1}, \cdots \gamma_{k}\right)}\left|f_{(m)(n)}\left(\gamma_{0}, \cdots \gamma_{k}\right)\right| .
\end{aligned}
$$

We have that

$$
\begin{gathered}
\sum_{\gamma_{k}: \gamma_{k} \cap\left(b \cup \ldots \cup \gamma_{k-1}\right) \neq \emptyset}\left|f_{(m)(n)}\left(\gamma_{0}, \gamma_{1}, . . \gamma_{k}\right)\right| \\
\leq 2\left[k+\left(\left|\gamma_{k}\right|+. .\left|\gamma_{k-1}\right|-k+1\right) v_{k}^{-1}\right] \\
\qquad \sup _{c \in \bar{\Lambda}} \sum_{\substack{\gamma_{k} \ni c \\
\left|\gamma_{(m)(n)}\left(\gamma_{0}, \gamma_{1}, \ldots \gamma_{k-1}\right)\right|}}\left|r_{m_{k} n_{k} \gamma_{k} \mid}\right| \\
\left|\gamma_{k}\right|=v_{k}
\end{gathered}
$$

The first term originates from the $k$ centers of noncommutativity of $\gamma_{0} \cup \gamma_{1} \ldots \cup \gamma_{k-1}$, while the second is due to the center of noncommutativity in $\tau_{\gamma_{k}}$. The factor $v_{k}^{-1}=$ $\left|\gamma_{k}\right|^{-1}$ is inherited from (2.14). Since the volumes are ordered, we have

$$
\left(\left|\gamma_{1}\right|+. .+\left|\gamma_{k-1}\right|-k+1\right) v_{k}^{-1} \leq k .
$$

Iterating this bound, we find

$$
\begin{aligned}
& \sup _{x} \sum_{k \geq 0} \sum_{\gamma_{0} \ni x} \sum_{n_{1}+\cdots+n_{k}=n-1} \sum_{\left|\gamma_{1}\right|=v_{1}} \frac{1}{n\left(v_{1}, \cdots v_{k}\right)}\left|f\left(b, \gamma_{1}, \cdots \gamma_{k}\right)\right| \\
& m_{1}+\cdots+m_{k}=m \quad \ldots \\
& \left|\gamma_{k}\right|=v_{k} \\
& \leq \sum_{k \geq 0}(4 \cdot k) \cdot(4 \cdot(k-1)) \cdots 4 \cdot \sum_{\substack{n_{1}+\cdots+n_{k}=n-1 \\
m_{1}+\cdots m_{k}=m}} \prod_{j=1, \cdots k} \sum_{\left|\gamma_{j}\right|=v_{j}}\left|r_{m_{j} n_{\jmath} \gamma_{\jmath}}\right| \\
& \leq \sum_{k \geq 0} 2^{2 k} k ! \sum_{\substack{n_{1}+\cdots+n_{k}=n \\
m_{1}+\cdots+m_{k}=m-1}} \prod_{\substack{\jmath+\cdots k \\
m_{j}, \cdots n_{j} v_{j}}} r^{*}
\end{aligned}
$$

where 


$$
r_{m_{j} n_{j} v_{j}}^{*}=\sup _{x} \sum_{\substack{\gamma \ni b \\\left|\gamma_{j}\right|=v_{j}}}\left|r_{m_{\jmath} n_{j} \gamma_{j}}\right| .
$$

Similarly, we find that the first three terms in (3.2) give a contribution to $r_{n}^{*}$ which is

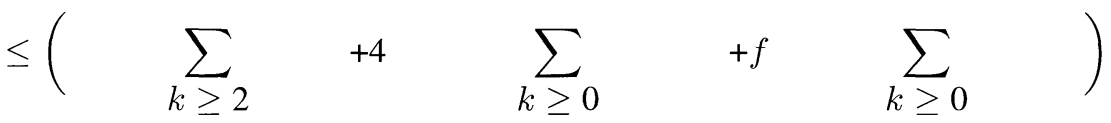

$$
\begin{aligned}
& n_{1}+\ldots+n_{k}=n \quad n_{1}+\ldots+n_{k}=n \quad n_{1}+\ldots+n_{k}=n-1 \\
& m_{1}+\ldots+m_{k}=m \quad m_{1}+\ldots+m_{k}=m-1 \quad m_{1}+\ldots+m_{k}=m \\
& \sum_{v_{1} \leq \ldots \leq v_{k}} \frac{2^{2 k} k !}{n\left(v_{1}, \ldots v_{k}\right)} \prod_{j=0, \ldots k} r_{m_{j} n_{j} v_{\jmath}}^{*}
\end{aligned}
$$

where $f=\sum_{x}\left\|f_{x}\right\|$. Using the binomial formula to compute the sum over the volumes, one can express these bounds in terms of the formal power series in (3.7) and find

$$
\begin{aligned}
r^{*}(t ; \lambda, \tau) \leq \lambda & +f \tau^{-1}+\left[\left(1-4 r^{*}(t ; \lambda, \tau)\right)^{-1}-1-4 r^{*}(t ; \lambda, \tau)\right] \\
& +\left(4 \lambda+(1+f) \tau^{-1}\right)\left[\left(1-4 r^{*}(t ; \lambda, \tau)\right)^{-1}-1\right]
\end{aligned}
$$

The analyticity of the function $r^{*}(t ; \lambda, \tau)$ is a consequence of the implicit function theorem and the lemma in Sect. 2 follows.

\section{References}

[A] Albanese, C.: Commun. Math. Phys. 134, 1-27 (1990); Commun. Math. Phys. 134, 237-272 (1990) [AF] Albanese, C., Froehlich, J.: ETH-TH preprint, 1993

[BF] Born, M., Fock, V.: Z.Phys. 51, 165-169 (1928)

[F] Forster, D.: Hydrodynamic Fluctuations, Broken Symmetry, and Correlation Functions. Reading, MA: Addison-Wesley, 1975

[GJ] Glimm, J., Jaffe, A.: Quantum Physics, a Functional Integral Point of View. Berlin, Heidelberg, New York: Springer, 1987

[K] Kato, T.: J. Phys. Soc. J. Jpn. 5, 435 (1950)

[JP] Joye, A., Pfister, C.: Commun. Math. Phys. 140, 15 (1991)

Communicated by Ya. G. Sinai

This article was processed by the author using the Springer-Verlag TEX PJour1g macro package 1991. 